\title{
Nuevos registros de Pyrenomycetes (Ascomycotina) en MÉXICO
}

\author{
Rosario Medel \\ Instituto de Ecología, A.C; Apartado Postal 63; 91000, Xalapa, Veracruz, México. \\ Correo electrónico: medelr@ecologia.edu.mx
}

\begin{abstract}
Resumen: Se discuten 20 especies de Pyrenomycetes de México, de los cuales 18 son Xylariales, uno es Hypocreal y uno Sordarial. Todos son nuevos registros para los estados de Baja California, Chiapas, Colima, Durango, Guerrero, Jalisco, Morelos, Nuevo León, Oaxaca, Quintana Roo, Sinaloa, Tabasco, Tamaulipas, Veracruz y Yucatán. Además, Podostroma alutaceum y Camillea cyclops son nuevos registros para México, de Chiapas y Veracruz, respectivamente.

Palabras clave: Pyrenomycetes, Xylariales, Sordariales, Hypocreales

Abstract: Twenty species of Pyrenomycetes from Mexico are discussed, 18 of which are Xylariales, one is a Hypocreal and one a Sordarial. All are new records from the following Mexican states: Baja California, Chiapas, Colima, Durango, Guerrero, Jalisco, Morelos, Nuevo León, Oaxaca, Quintana Roo, Sinaloa, Tabasco, Tamaulipas, Veracruz, and Yucatán. Podostroma alutaceum and Camillea cyclops are new records for Mexico, from Chiapas and Veracruz, respectively.
\end{abstract}

Key words: Pyrenomycetes, Xylariales, Sordariales, Hypocreales

E 1 conocimiento de los ascomicetes en México es aún escaso. De los más de 32,000 especies conocidas para este grupo (Hawksworth et al., 1995), sólo se habían citado para México, hasta 1996, 544 especies; de éstas, aproximadamente $46 \%$ son Pyrenomycetes (Medel et al., 1999). Sin embargo, dado el número de especies existentes, este porcentaje es mínimo, por lo que es necesario continuar con el inventario de ascomicetes mexicanos. Por otro lado, recientemente Medel et al. (1999) hicieron ver que hacen falta estudios sobre estos hongos, especialmente en la zona norte, noreste y sureste del país, regiones donde los registros de estos hongos son escasos o nulos. De esta manera, el objetivo del presente trabajo es continuar con el inventario sobre ascomicetes mexicanos, presentando nuevos registros de Pyrenomycetes, procedentes de 15 entidades del país, a saber: Baja California (1), Chiapas (2), Colima (2), Durango (2), Guerrero (1), Jalisco (2), Morelos (2), Nuevo León (2) Oaxaca (1), Quintana Roo (1), Sinaloa (1), Tabasco (3), Tamaulipas (1), Veracruz (4) y Yucatán (3).

Algunos de los géneros tratados en este trabajo ya han sido estudiados anteriormente en México, como es el caso de Camarops (San Martín y Lavín, 1997), Daldinia (Pérez-
Silva, 1973), Hypoxylon (Pérez-Silva, 1983; Medel et al., 1989; San Martín et al., 1999) y Poronia (Pérez-Silva, 1970).

\section{Materiales y métodos}

En el estudio microscópico de los especímenes se emplearon $\mathrm{KOH} 5 \%$, azul de algodón y solución de Melzer. En la descripción de colores de la especie Podostroma alutaceum (Pers.: Fr.) Atk., se utilizó el manual de Kornerup y Wanscher (1978). Todo el material revisado se encuentra depositado en el Herbario del Instituto de Ecología (XAL); algunos son duplicados de los siguientes herbarios: Universidad Autónoma Metropolitana Iztapalapa (UAMIZ), Escuela Nacional de Ciencias Biológicas del Instituto Politécnico Nacional (ENCB), Instituto de Botánica de la Universidad de Guadalajara (IBUG) y Universidad Autónoma de Baja California en Ensenada (BCMEX). Para la identificación de las especies se utilizaron obras especializadas como las de Dennis (1957, 1970, 1978), Miller (1961), Ju y Rogers (1996), Ju et al. (1997) y San Martín y Lavín (1997), principalmente. Para el caso de las especies que ya se han citado en otros trabajos, se omiten las descripciones completas, pero se 


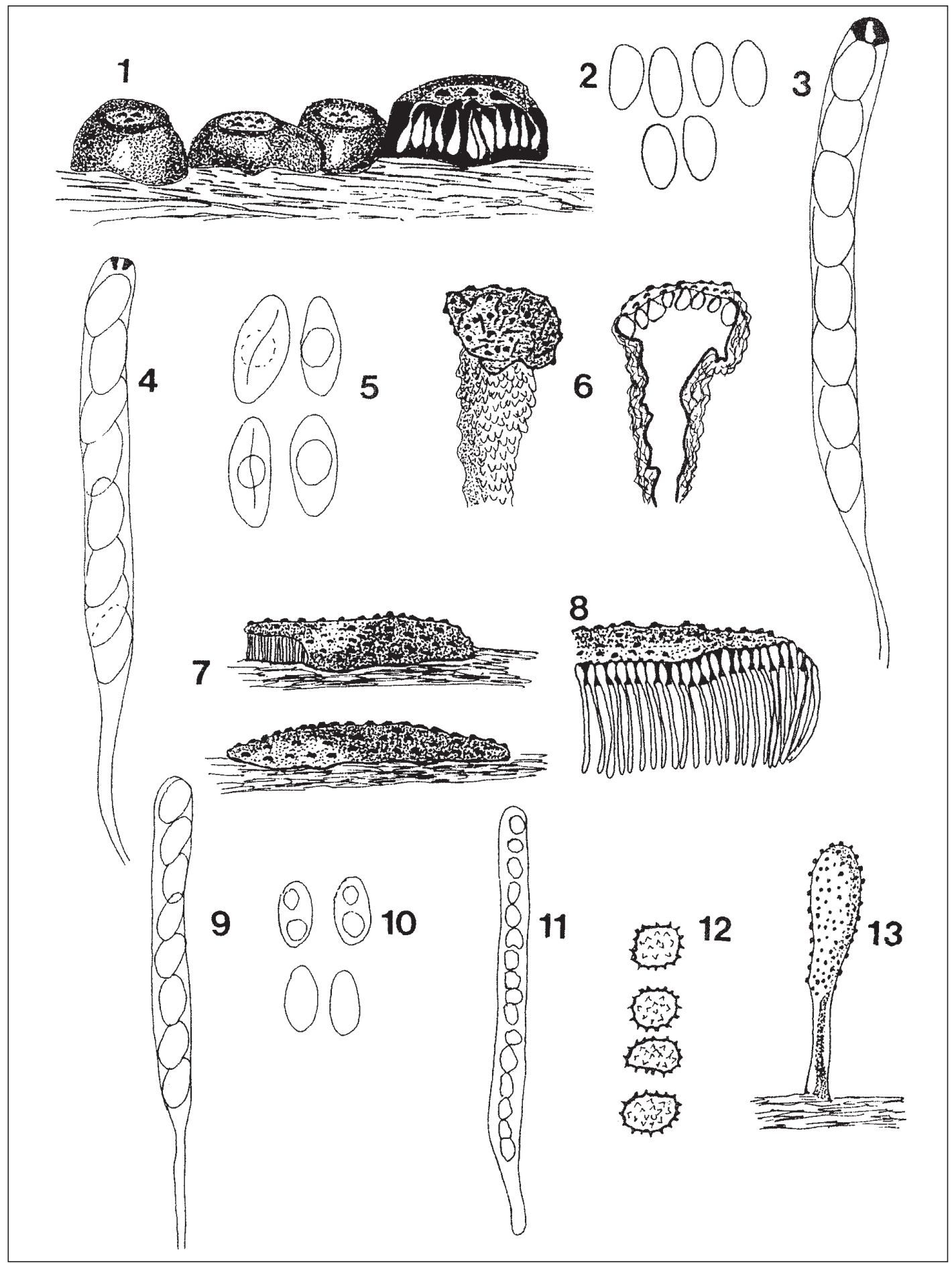

Figuras 1-13. 1-3. Camillea cyclops. 1: estromas, 2: ascosporas, 3: ascas con ascosporas. 4-6. Kretszchmaria heliscus. 4: asca con ascosporas, 5: ascosporas, 6: estroma y corte transversal del mismo. 7-10. Camarops polyspermun, 7: estromas, 8: corte transversal del estroma mostrando los peritecios tubulares, 9: asca con ascosporas, 10: ascosporas. 11-13. Podostroma alutaceum. 11: asca con ascosporas, 12: ascosporas, 13: estromas. 
anotan algunas características diferenciales en cada caso y se discute la distribución geográfica. Únicamente se describen e ilustran aquellas especies poco conocidas o nuevos registros.

\section{Especies estudiadas}

Xylariales

Camillea cyclisca (Mont.) Laessøe, J.D. Rogers \& Whalley, Myc. Res. 93:121.

Los estromas aplanados, con peritecios pequeños de 0.3 $\mathrm{mm}$ de diámetro y ascosporas de 14-24 x 5-7.5 $\mu \mathrm{m}$, caracterizan a esta especie (Miller, 1961; Laessøe et al., 1989). Morfológicamente esta especie es afín a C. punctulata (Berk. \& Ravenel) Laessøe, J.D. Rogers \& Whalley, una especie que se conoce también de México de bosque mesófilo de montaña y bosque de pino encino (Medel et al., 1989; San Martín y Rogers, 1993b).

Hábitat. Lignícola, en bosque tropical perturbado.

Material estudiado. Yucatán: zona $\mathrm{N}$ de las ruinas de Uxmal, S. Chacón 2728 (XAL).

Discusión. Esta especie se conocía de Chiapas y Tamaulipas (San Martín y Rogers, 1993b); de acuerdo con Laessøe et al . (1989), se distribuye en Costa Rica, Ecuador, Guyana Francesa y Perú.

Camillea cyclops (Mont.) Berk. \& M.A. Curtis, J. Acad. Nat. Sci. Philadelphia, N.S. 2: 285. 1853.

Figs.1-3

Estroma errumpente, cilíndrico, más ancho que alto, de 5-7 $\times 2.5 \mathrm{~mm}$, con un disco apical plano, rodeado por un borde ancho, carbonoso, negro. Peritecios numerosos, alargados, hasta de $2 \mathrm{~mm}$ de longitud, los cuales se unen a un canal estrecho que termina en un ostiolo papilado en el centro de un disco apical plano. Ostiolos papilados de 2-7 en cada disco. Ascas cilíndricas de 144-160 x 8-8.8 $\mu \mathrm{m}$, estipitadas, con anillo apical fuertemente amiloide en Melzer, octoporados. Esporas elípticas, más estrechas hacia la base de la espora, de 9.6-10.4 x $4.8(-5.6) \mu \mathrm{m}$, de color amarillo pálido a café claro. Paráfisis filiformes, delgadas, abundantes.

Hábitat. Lignícola, selva alta perennifolia perturbada.

Material estudiado. Veracruz: brecha sur en el Poblado 2, Región de Uxpanapan, municipio de Jesús Carranza, López 1033 (ENCB, XAL).

Discusión. El estroma cilíndrico más ancho que alto, la presencia de canales que se abren hacia un anillo de ostiolos sobre un disco plano y la medida de las esporas caracterizan a esta especie, de acuerdo con Dennis $(1957,1970)$ y Laessøe et al. (1989). C. cyclops se distribuye en la Guyana Francesa, Colombia, Panamá y Venezuela (Laessøe et al., 1989); éste es el primer registro para México. Con este registro suman ya 17 especies de Camillea citadas para el país.

Daldinia cudonia (Berk. \& M.A. Curtis) C.G. Lloyd, Mycol. Writings 7: 1255. 1924.
Esta especie se caracteriza por el estroma turbinado a clavado, de color café-vináceo con el estípite delgado y pequeño, además de la medida de las ascosporas de 12.2$16.6(-19.5) \times 6-8 \mu \mathrm{m}$ (Ju et al., 1997). Es una especie afín a D. loculata (Lév.) Sacc., la cual fue registrada también del estado de Veracruz.

Hábitat. Lignícola, en bosque mesófilo de montaña.

Material estudiado. Jalisco: El Laurelito, predio Las Joyas, Sierra de Manantlán, municipio de Autlán, Guzmán 29059 (XAL).

Discusión. Esta especie está registrada de México y porciones más boreales de Norteamérica; para México fue citada de Veracruz, de bosque mesófilo de montaña (Pérez-Silva, 1973; Ju et al., 1997).

Daldinia eschscholzii (Ehrenb.: Fr.) Rehm, Ann. Mycol. 2: 175. 1904.

El estroma turbinado, sésil o con un corto estípite, el color café vináceo a púrpura, así como la medida de las esporas (10-14 (-15.5) x 5-6.5 $\mu \mathrm{m})$, caracterizan a esta especie, según Ju et al. (1997).

Hábitat. Lignícola, en matorral xerófilo.

Material estudiado. Nuevo León: Lampasos, matorral con dominancia de Prosopis y Acacia, F. Garza 39 (ENCB, XAL); Veracruz: alrededores de El Grande, municipio de Coatepec, cafetal, Jarvio 749 (XAL); Yucatán: Carretera Mérida a Tixkokob, 2 km antes de la desviación de Oncán, Guzmán 24737 (XAL).

Discusión. Éste es un hongo de distribución amplia en América, Asia y Australia; de México se ha citado de los estados de Campeche, Oaxaca y Quintana Roo, de vegetación tropical (Ju et al., 1997).

Daldinia grandis Child, Ann. Missouri Bot. Gard. 19: 456. 1932.

Ésta se caracteriza por el estroma hemisférico, sésil o casi sésil y por el tamaño tanto del estroma $(2.5-8 \times 1.5-5.5 \mathrm{~cm})$ como de las ascosporas (14-22 (-25.5) x 7-11 $\mu \mathrm{m})$, de acuerdo con Ju et al. (1997).

Hábitat. Lignícola, en bosque de Pinus.

Material estudiado. Morelos: Monumento a Morelos, carretera México a Cuernavaca, bosque de Pinus spp., Guzmán 11417 (ENCB, XAL); Veracruz: cerca del Ingenio el Rosario, vertiente E del Cofre de Perote, municipio de Xico, Medel 767 (XAL).

Discusión. Esta especie se conoce de Nueva Zelanda, Estados Unidos, y de México anteriormente fue citada de Nuevo León, Tamaulipas y Baja California, de bosques de encinos (Ju et al., 1997; Medel y Chacón, 1992).

Hypoxylon archeri Berk. apud J.D. Hook., Bot. Antarc. Voy. II. pt.II.p280. 1860.

La forma del estroma, los pigmentos verde-oliváceo obscuros y los ostiolos papilados en medio de un disco 
pequeño menor de $0.1 \mathrm{~mm}$ (tipo Hypoxylon truncatum), caracterizan a esta especie, de acuerdo con Miller (1961) y Ju y Rogers (1996).

Hábitat. Lignícola, en selva baja subperennifolia y zona agrícola.

Material estudiado. Quintana Roo: Estación Experimental del CIQRO, $3 \mathrm{~km}$ al $\mathrm{N}$ de Puerto Morelos, carretera a Cancún, López 1603 (XAL).

Discusión. Se distribuye en América y Asia (Ju y Rogers, 1996); de México fue citada de Chiapas, Hidalgo y Veracruz por Medel et al. (1989), de vegetación tropical y bosque mesófilo de montaña.

Hypoxylon cinnabarinum (Henn.) Y.-M. Ju \& J. D. Rogers, A revision of the genus Hypoxylon, p. 99. 1996.

$=$ H. rubiginosum var. tropica $\mathrm{J}$. H. Miller.

Esta especie se caracteriza por el color ferruginoso a rojo púrpura y por el tamaño de las ascosporas (9.5-14 x 5-7.5 $\mu \mathrm{m})$, de acuerdo con Miller (1961) y Ju y Rogers (1996).

Hábitat. Lignícola, en bosque de Ilex socorroensis.

Material estudiado. Colima: Archipiélago Revillagigedo, Isla Socorro, A. Flores 785 (XAL).

Discusión. Esta especie se distribuye principalmente en los trópicos (Miller, 1961); de México fue citada de Tabasco (Ju y Rogers, 1996) y de Jalisco (Guzmán-Dávalos et al., 1983), donde crece en vegetación tropical.

Hypoxylon crocopeplum Berk. \& M. A. Curtis apud Berk., Grevillea 4:49. 1875.

La especie se distingue por los peritecios semiglobosos a oblongos y por la medida de las esporas de 9.5-15 (-17) x 47 (-7.5) ㅆm, según Ju y Rogers (1996) y Miller (1961). Es afín a $H$. haematostroma Mont., especie también presente en México (Ju y Rogers, 1996), pero esta última presenta peritecios tubulares y esporas más grandes, hasta de $18 \mu \mathrm{m}$. Hábitat. Lignícola, en bosque tropical.

Material estudiado. Oaxaca: plantación de pinos de la fábrica de papel Tuxtepec, G. Betancourt 175 (ENCB, XAL).

Discusión. Esta es una especie de amplia distribución en los trópicos y subtrópicos del país; ha sido citada de Nayarit (Martin, 1969), Quintana Roo y Tamaulipas (Ju y Rogers, 1996).

Hypoxylon haematostroma Mont. apud Sagra, Hist. Phys. Pol. Nat. Cuna IX, p. 344. 1845.

El color naranja rojizo del estroma, los peritecios tubulares, así como el tamaño de las esporas (13.5-18(-19) x 7-9 $\mu \mathrm{m})$ caracterizan a esta especie (Miller, 1961; Ju y Rogers, 1996). Hábitat. Lignícola en vegetación tropical.

Material estudiado. Colima: cerca del hotel Playa Santiago, Bahía de Santiago, NO de Manzanillo, G. Guzmán 6219 (ENCB, XAL); Guerrero: Cascadas de las Granadas, Guzmán-Dávalos 75 (ENCB, XAL).

Discusión. De acuerdo con Miller (1961) y Ju y Rogers (1996), ésta es una especie de distribución tropical. Para México anteriormente fue citada de Campeche, Chiapas, Morelos, Oaxaca, Quintana Roo, Tamaulipas, Veracruz y Yucatán (Guzmán, 1983; Medel et al., 1989; Ju y Rogers, 1996; San Martin et al., 1999).

Hypoxylon hypomiltum Mont., Ann. Sci. Nat. Bot. sér. II, 13:356. 1840.

Esta especie se caracteriza por el color naranja rojizo del estroma y, a diferencia de la especie anterior, ésta presenta peritecios oblongos o semiglobosos y esporas más pequeñas, de (6.5-) 7-9 x 3-4 $\mu \mathrm{m}$ (Miller, 1961; Ju y Rogers, 1996).

Hábitat. Lignícola, en bosque espinoso.

Material estudiado. Sinaloa: El Portezuelo, carretera Mazatlán a Tepic, G. Rodríguez 02691 (UAMIZ, XAL).

Discusión. H. hypomyltum es una especie de distribución netamente tropical; se conocía de México de los estados de Quintana Roo (Guzmán, 1983) y Tabasco (Ju y Rogers, 1996), donde crece en vegetación tropical.

Hypoxylon lenormandii (Berk. \& M.A. Curtis) apud Berk., J. Linn. Soc., Bot. 10: 385. 1869.

De acuerdo con Ju y Rogers (1996), la especie se distingue por el estroma efuso que tiende a ser peritecioide, por el color gris-sepia a café vináceo, los pigmentos naranja-ferruginoso, ámbar a ocráceos extraídos en $\mathrm{KOH} 10 \%$, y por el tamaño de las esporas $(9.5-15(-16) \times 4-6.5 \mu \mathrm{m})$.

Hábitat. Lignícola, en acahual.

Material estudiado. Yucatán: carretera Mérida a Tixkokob, 2 km antes de la desviación a Oncán, Chacón 2706 (XAL).

Discusión. Esta especie es de amplia distribución en América, Asia y África; crece en una amplia variedad de hospederos ya sea mono o dicotiledóneas. Anteriormente fue citada de Chiapas, Nuevo León (Ju y Rogers, 1996), Sinaloa, Nayarit (Martin, 1969, como H. oodes Berk \& Broome) y por San Martín et al. (1999) de Campeche y Tamaulipas, donde crece en vegetación tropical.

Hypoxylon notatum (Berk. \& M. A. Curtis) apud Berk., Grevillea 4: 50. 1875.

Los peritecios en glomérulos y el color café claro del estroma, así como el tamaño de las ascosporas de 12-16 x 6.5-7.5 $\mu \mathrm{m}$ (Miller, 1961; Ju y Rogers, 1996) distinguen a esta especie.

Hábitat. Lignícola, en bosque tropical.

Material estudiado. Tabasco: playa Las Pomarrosas, ejido Central Fournier, Laguna del Rosario, municipio de Huimanguillo, G. Rodríguez 981 (XAL).

Discusión. Es una especie de distribución amplia, asociada principalmente a diferentes especies de Quercus, de acuerdo con Ju y Rogers (1996). H. notatum fue citado por Medel et al. (1989) del estado de Veracruz, de bosque mesófilo de montaña. 
Hypoxylon rubiginosum (Pers.: Fr.) Fr., Summa Veg. Scand. II p. 384. 1849.

La especie se caracteriza por el color café-rojizoferruginoso que presenta el estroma y por el tamaño de las esporas de 9-12.8 x 4-6 $\mu \mathrm{m}$, además de los pigmentos color naranja-rojizo extraídos en KOH 10\% (Ju y Rogers, 1996). Hábitat. Lignícola, en bosque tropical deciduo.

Material estudiado. Morelos: $3 \mathrm{~km}$ al S de Amador Salazar, Cañón de Lobos, antigua carretera México a Cuautla, Guzmán 11382 (ENCB, XAL); Tabasco: Colegio Superior de Agricultura Tropical, km 21 carretera Cárdenas a Coatzacoalcos, Solano 208 (XAL).

Discusión. Ésta es una especie cosmopolita que fue citada de Puebla, Sonora, Quintana Roo, Veracruz y Yucatán (Martínez-Alfaro et al., 1983; Welden y Guzmán, 1978; Pérez-Silva et al., 1996), de vegetación tropical y bosque mesófilo de montaña.

Hypoxylon thouarsianum (Lév.) Lloyd, Mycol. Writings 5: 26. 1919.

La forma globosa a hemisférica del estroma, así como los ostiolos papilados en medio de un disco hundido y elevado en el margen, además del tamaño de las esporas (14-24 x 4$5.5 \mu \mathrm{m}$ ), distinguen a esta especie (Miller, 1961; Ju y Rogers, 1996).

Hábitat. Lignícola, en bosque de encinos.

Material estudiado. Baja California: Santa Rosa, carretera Ensenada a Tijuana, A. Álvarez 12, El Junco, $15 \mathrm{~km}$ al NO de Ensenada, Ayala 26 (BCMEX, XAL).

Discusión. Este hongo parece estar confinado a África y América (Ju y Rogers, 1996) y es una especie que se distribuye ampliamente en México (Pérez-Silva, 1983), en 14 entidades del país y en casi todos los tipos de vegetación (Medel et al., 1989; San Martín et al., 1999).

Hypoxylon truncatum (Schwein : Fr.) J. H Miller, Trans. Brit. Mycol. Soc. 17: 130. 1823.

Esta especie se caracteriza por los ostiolos papilados en medio de un disco aproximadamente de hasta $4 \mathrm{~mm}$ de diámetro, por los pigmentos de color verde oliváceo extraídos en $\mathrm{KOH} 10 \%$ y por el tamaño de las esporas (8-10.5 x 4-5 $\mu \mathrm{m})$, de acuerdo con Miller (1961) y Ju y Rogers (1996).

Hábitat. Lignícola, en selva alta perennifolia, bosque de Pinus y bosque espinoso.

Material estudiado. Chiapas: río Chancalá, municipio de Chancalá, Chacón 3351 (XAL); Durango, 20-30 km al sur de Piedra Herrada, Reserva de la Biosfera de La Michilía, $G$. Rodríguez 626 (UAMIZ, XAL); Tamaulipas: km 68 carretera Cd. Victoria a Soto la Marina, Valenzuela 1513 (ENCB, XAL). Discusión. Esta es una especie de amplia distribución, principalmente en el trópico (Miller, 1961). De México fue citada anteriormente de Nuevo León y Quintana Roo (Ju y Rogers, 1996), pero sin localidad precisa (Miller, 1961); se registra por primera vez para Chiapas, Durango y Tamaulipas.
Poronia oedipus Mont., Ann. Sci. Nat. Bot. Ser. 4, 3: 114. 1855.

Este hongo se caracteriza por la forma del estroma, el cual presenta un estípite largo y por el tamaño pequeño de las esporas de 23-30 x 14-16 um (Dennis, 1957; Jong y Rogers, 1969).

Hábitat. Fimícola, en pastizal con Acacia y bosque de pinoencino.

Material estudiado. Durango: km 32 carretera Durango a Torreón, G. Rodríguez 790 (UAMIZ, XAL); Jalisco: Bosque de la Primavera, $2 \mathrm{~km}$ al suroeste del Motoautódromo, municipio de Zapopan, Guzmán-Dávalos 1614-B, Guzmán 24601 (XAL), S de Etzatlán, Sierra de la Mesa, O. Rodríguez 1803 (XAL, IBUG); Nuevo León: San Nicolás de los Garza, I. Nuñez 22 (XAL).

Discusión. Esta especie es de amplia distribución en México. Fue registrada de los estados de Chiapas, Guerrero, Oaxaca, Puebla, Veracruz (Pérez-Silva, 1970), Michoacán (DíazBarriga et al., 1988), Hidalgo (Frutis y Guzmán, 1983), Tamaulipas (San Martín y Rogers, 1993a) y Sonora (PérezSilva et al., 1996), de bosque de pinos, potreros, bosque mesófilo de montaña y bosque de encinos.

Poronia punctata (L.) Fr., Summa Veg. Scand. Sect. Post. P. 382. 1849.

La especie se caracteriza por la forma discoide de la parte fértil del estroma, además de presentar esporas más pequeñas (22-28 × 6-8 $\mu \mathrm{m})$ que las de la especie anterior (Munk, 1957; Pérez-Silva, 1970).

Hábitat. Fimícola, en bosque mesófilo de montaña.

Material estudiado. Morelos: antigua carretera México a Cuernavaca, desviación a Fierro del Toro, Guzmán 287 (ENCB, XAL), Santa Catarina, El Texcal, carretera Tepoztlán a Cuernavaca, Guzmán 18137 (ENCB, XAL).

Discusión. Es una especie de distribución amplia en América y Europa (Munk 1957; Dennis, 1970). Anteriormente fue citada del Distrito Federal, el Estado de México (Pérez-Silva, 1970) y Durango (Pérez-Silva y Aguirre-Acosta, 1985).

Xylaria heliscus (Mont.) J. D. Rogers \& Y.-M. Ju

= Kretzschmaria heliscus (Mont.) Massee

Figuras 4-6

El estroma clavado, ramificado desde la base, con la parte fértil en forma de cabezas globosas y el tamaño de las esporas (11-13 x 5-6 $\mu \mathrm{m})$ son características de esta especie, de acuerdo con San Martín y Rogers (1993a) y Rogers et al., (1987). Recientemente Rogers y Ju (1998) hicieron ver que a pesar de que la especie presentaba estroma de tipo Kretschmaria Fr., el hecho de que el endostroma no llegue a ser hueco con la edad, la capa estromática exterior sea delgada, no carbonosa y las ascosporas sean pequeñas (9-10 x 5-6 $\mu \mathrm{m}$ ), sugieren un mejor acomodo dentro del género Xylaria Hill. ex Schrank. 
Hábitat. Lignícola, en plantación de cacao.

Material estudiado. Tabasco: San Antonio Cunduacán, Solano 72 (XAL).

Discusión. Esta especie fue citada de Chiapas (Medel y Chacón, 1992) y de Veracruz por San Martín y Rogers (1993a) como Kretzschmaria heliscus. Se distribuye ampliamente en el trópico y el subtrópico; ésta es la primera cita para el estado de Tabasco.

\section{Sordariales}

Camarops polyspermun (Mont.) J.H. Miller, Trans. Brit. Mycol. Soc. 15: 151. 1930.

Figuras 7-10

Esta especie se caracteriza por el estroma en forma de almohadilla y los peritecios largamente cilíndricos (Dennis, 1970, 1978; Miller, 1930); sin embargo, existe un carácter no citado en la literatura que fue anotado por el colector: la secreción de un líquido negro, conspicuo. Fue citada de México por San Martín y Lavín (1997) de San Luis Potosí, de selva baja caducifolia.

Hábitat. Lignícola, en cafetal relicto de bosque mesóflo de montaña.

Material estudiado. Veracruz: Zoncuantla, municipio de Coatepec, cerca de la carretera antigua Xalapa a Coatepec, Guzmán 30893 (XAL).

Discusión. Camarops es un género que se distribuye tanto en Europa como en América (Dennis 1970, 1978). La especie fue citada de México por San Martín y Lavín (1997) de San Luis Potosí, de selva baja caducifolia, y en esta ocasión se cita por vez primera para Veracruz.

\section{Hypocreales}

Podostroma alutaceum (Pers.: Fr.) Atk., Bot. Gaz. 40: 416. 1905.

Figuras 11-13

Estroma vertical, clavado, formado por un pie estéril y una cabezuela más o menos irregular de color naranja (5B8) a café amarillento (5C8) en seco, con la parte interna de color amarillo pálido (3A3). La parte fértil de apariencia irregular rugosa a equinulada, debido a los ostiolos de los peritecios que están total o parcialmente inmersos en el estroma, 27 $\mathrm{mm}$ de longitud $\times 4 \mathrm{~mm}$ de diámetro, más delgado hacia el pie ( $2 \mathrm{~mm}$ diámetro). Ascas cilíndricas o ligeramente clavadas, cortamente estipitadas, de 64-72 $\times 4-4.8 \mu \mathrm{m}$, inamiloides, con 16 esporas. Esporas de 3.2-4-(4.8) x 3.2 $(-4) \mu \mathrm{m}$, hialinas, ligeramente equinuladas. No se observaron paráfisis.

Hábitat. Lignícola, en selva alta perennifolia.

Material estudiado. Chiapas: camino a las ruinas Lacanjá, Municipio de Ocosingo, Chacón 2014 (XAL).

Discusión. El material estudiado concuerda con las descripciones de Seaver (1910), Dennis (1978) y Breitenbach y Kränzlin (1981), excepto que se menciona el estroma de color amarillo pálido, blanquecino o de color marrón, diferente al citado en el material mexicano. La especie se ha asociado a bosques de coníferas (Dennis, 1978) y árboles de hojas anchas (Breitenbach y Kränzlin, 1981). Dennis (1970) mencionó esta especie para Venezuela, aunque anotó que el material estaba inmaduro. Se cita por primera vez de México del estado de Chiapas.

\section{Conclusiones}

De las 20 especies citadas, 18 son Xylariales, lo cual concuerda con el dato que enfatizó Whalley (1993) de que aproximadamente $75 \%$ de los Xylariales se encuentran en zonas tropicales. Por otro lado, las especies encontradas se distribuyeron en diversos tipos de vegetación, a saber: vegetación tropical (16), bosque mesófilo de montaña (3), bosque de pino (2), bosque de encino (1), y bosque de pinoencino (1), mostrando con esto que los Pyrenomycetes son un grupo de gran diversidad, que se encuentra en una gran variedad de hábitats. En este trabajo se incrementan los registros de Pyrenomycetes para estados con muy pocos o nulos registros, como es el caso de Baja California, Colima, Sinaloa y Yucatán, entidades de las que se habían citado 6, 6, 10 y 10 especies de hongos Ascomicetes, respectivamente, de acuerdo con Medel et al. (1999).

\section{Agradecimientos}

La autora agradece al Dr. Francisco Lorea del Instituto de Ecología la revisión crítica del trabajo. A Juan Lara y Ma. Eugenia Ramírez, de la misma Institución, se les agradece su ayuda en diversas tareas. Al M. en C. Ricardo Valenzuela (ENCB), Dra. Nahara Ayala (BCMEX), M. en C. Laura Guzmán-Dávalos (IBUG), y Biól. Guillermo Rodríguez (UAMIZ) se les agradece haber proporcionado duplicados de los materiales estudiados. A los revisores anónimos que contribuyeron a mejorar sustancialmente el trabajo.

\section{Literatura citada}

Breitenbach J. y Kränzlin F. 1981. Fungi of Switzerland, Vol. I. Ascomycetes. Verlag Mykologia, Lucerna.

Dennis R.W.G. 1957. Further notes on tropical American Xylariaceae. Kew Bulletin 12:297-332.

Dennis R.W.G. 1970. Fungus Flora of Venezuela and Adjacents Countries. J. Cramer, Vaduz.

Dennis R.W.G. 1978. British Ascomycetes. J. Cramer, Vaduz.

Díaz-Barriga H., Guevara-Fefer F. y Valenzuela R. 1988. Contribución al conocimiento de los macromicetos del estado de Michoacán. Acta Botanica Mexicana 2:21-44.

Frutis I. y Guzmán G. 1983. Contribución al conocimiento de los hongos del estado de Hidalgo. Boletín de la Sociedad Mexicana de Micología 18:219-266.

Guzmán G. 1983. Los hongos de la Península de Yucatán II. Nuevas exploraciones y adiciones micológicas. Biótica 8:71-100.

Guzmán-Dávalos L., Nieves G. y Guzmán G. 1983. Hongos del 
estado de Jalisco II. Especies depositadas en el herbario ENCB. Boletín de la Sociedad Mexicana de Micología 18:125-158.

Hawksworth D.L., Kirk P.M., Sutton B.C. y Peg D.N. 1995. Ainsworth \& Bisby's Dictionary of the Fungi. 8a ed. Cab International, Cambridge.

Jong S.C. y Rogers J.D. 1969. Poronia oedipus in culture. Mycologia 61:853-862.

Ju Y.-M. y Rogers J.D. 1996. A revision of the genus Hypoxylon. Mycologia Memoir 20. APS Press, St. Paul, Minnesota.

Ju Y.-M., Rogers J.D. y San Martin F. 1997. A revision on the genus Daldinia. Mycotaxon 61:243-294.

Kornerup A. y Wanscher J.H. 1978. Methuen Handbook of Colour. 3a. ed., Methuen, Londres.

Laessøe T., Rogers J. D. y Whalley A.J.S. 1989. Camillea, Jongiella and light spored species of Hypoxylon. Mycological Research 93:121-155.

Martin P. 1969. Studies in the Xylariaceae V. Euhypoxylon. Journal of South African Botany 35:149-206.

Martínez-Alfaro M.A., Pérez-Silva E. y Aguirre-Acosta E. 1983. Etnomicología y exploraciones micológicas en la Sierra Norte de Puebla. Boletín de la Sociedad Mexicana de Micología 18:5163.

Medel R. y Chacón S. 1992. Ascomycetes lignícolas de México III. Algunos Sphaeriales. International Journal of Mycolgy and Lichenology 5:253-260.

Medel R., Chacón S. y G. Guzmán G. 1989. Especies conocidas y nuevos registros de Hypoxylon (Sphaeriales, Xylariaceae) en México. Revista Mexicana de Micología 5:149-168.

Medel R., Guzmán G. y Chacón S. 1999. Especies de macromicetos citadas de México IX. Ascomycetes, parte III: 1983-1996. Acta Botanica Mexicana 46:57-72.

Miller J.H. 1930. British Xylariaceae. Transactions of the British Mycological Society 15:134-154.

Miller J.H. 1961. A Monograph of the World Species of Hypoxylon. University of Georgia Press, Athens, Georgia.

Munk A. 1957. Danish Pyrenomycetes. Dansk Botanisk Arkiv Udgivet af Dansk Botanisk Forening 17:1-491.
Pérez-Silva E. 1970. Datos sobre el género Poronia en México. Boletín de la Sociedad Botánica de México 31:139-146.

Pérez-Silva E. 1973. El género Daldinia en México. Boletín de la Sociedad Mexicana de Micología 7:51-58.

Pérez-Silva E. 1983. Distribución de algunas especies del género Hypoxylon (Pyrenomycetes) en México. Anales del Instituto de Biología de la UNAM, Serie Botánica 54:1-22.

Pérez-Silva E. y Aguirre-Acosta E. 1985. Micoflora del estado de Durango, México. Revista Mexicana de Micología 1:315-329.

Pérez-Silva E., Esqueda-Valle M. y Armenta-Calderón A. 1996. Ascomycetes de Sonora I: Discomycetes y Pyrenomycetes. Revista Mexicana de Micología 12:97-106.

Rogers J.D., Callan B.E. y Samuels G.J. 1987. The Xylariaceae of the rain forest of north Sulawesi. Mycotaxon 29: 113-172.

Rogers J. D. y Ju Y.-M. 1998. The genus Kretzschmaria. Mycotaxon 68:345-393.

San Martín F. y Lavín P.A. 1997. Los ascomycetes Acanthonitschkia, Corynelia, Lopadostoma y Camarops en México. Acta Botanica Mexicana 41:31-41.

San Martín F. y Rogers J.D. 1993a. Kretzschmaria, Leprieuria y Poronia in Mexico. Mycotaxon 48:179-192.

San Martín F. y Rogers J.D. 1993b. Biscogniauxia and Camillea in Mexico. Mycotaxon 47:229-258.

San Martín F., Ju Y.-M. y Rogers J.D. 1999. Algunas especies de Hypoxylon (Pyrenomycetes, Xylariaceae) de México. Acta Botanica Mexicana 47:31-53.

Seaver F.J. 1910. The Hypocreales of North America III. Mycologia 2:48-92.

Welden A.L. y Guzmán G.. 1978. Lista preliminar de los hongos, líquenes y mixomicetos de las regiones de Uxpanapa, Coatzacoalcos, Los Tuxtlas, Papaloapan y Xalapa (parte de los estados de Veracruz y Oaxaca). Boletín de la Sociedad Mexicana de Micología 12:59-102.

Whalley A.J.S. 1993. Tropical Xylariaceae. Their distribution and ecological characteristics. En: Isaac S., Frankland J.C., Watling R. y Whalley A.J.S. Eds. Aspects of Tropical Mycology, pp.103120, Cambridge University Press, Cambridge. 